tion (Osprey process) for the production of bulk engineering components will be those that will mark the 1990 s.

It would be foolish to bet on any specific material, because the future will see the consolidation and integration of many current materials and technologies. If I were to stick my neck out, I think that there will be a new era for wood and other biological materials. Timber produced from microwave-treated wood has recently opened up a fresh vista for structural engineers. Silk and artificially grown cartilage are also areas where lateral thinking and interdisciplinary research are beginning to pay dividends. We can also hope to see materials education turn from a frightened remnant based on the steel industry to take a central role in the total design and production sequence. This may be despite the reactionary drag of the professional engineering institutions. There is a need for a coherent language that links the possibilities of scientific curiosity with the reality of engineering products. That link is to be found in the materials specialist, and it is time for the inferiority complex of the past two decades to be replaced by an optimism and sense of immediacy that the subject really deserves.

John Wood is currently Senior Lecturer in the Materials Discipline, The Open University, Walton Hall, Milton Keynes MK7 6AA, UK, but will shortly take up a post as Cripps Professor of Materials Engineering at the University of Nottingham.

\section{Special effects}

\section{F. Moriarty}

Ecotoxicology: Problems and Approaches. Edited by Simon A. Levin, Mark A. Harwell, John R. Kelly and Kenneth D. Kimball. Springer-Verlag: 1989. Pp.547. DM90, £29.50, \$49.

ECOTOXICOLOGISTs attempt to study the effects of chemicals on ecosystems. In the early days, about 25 years ago, we struggled to reject the use of $\mathrm{LD}_{50}$ tests and related measures, and to find more relevant ways of assessment. There was an important dichotomy of views in the underlying ecological science at that time. Clements had stated in 1916 that communities of interacting populations are organic entities comparable to individual organisms. Although Ramensky had published the first opposition as early as 1924, this view was widely accepted for the next 60 years. It is probably fair to say that the concept now has few adherents, but it still exerts a malign, usually covert, influence on the development of ecotoxicology.

In the second chapter of the book under review, J. R. Kelly and M. A. Harwell set the tone for much that follows by arguing at length that ecosystems are not supraorganisms and therefore one cannot expect to find one single or a few simple indicators of effects of exposures to chemicals. In particular the idea that functional indicators, such as rates of energy and of nutrient flow, are preferable to structural indicators, such as population size, is finally put to rest by several contributors. Kelly and Harwell stress that the choice of indicators of effects is a value judgement: in their terms, the choice should be "of relevance to issues of concern to humans". This statement deserves emphasis, particularly for what might be termed pure conservation interests - reasons why it is desirable to control impacts on wildlife are often fudged and obscured.
One might hope, after that start, for detailed examination of suitable indicators, but the opportunity is largely missed. Thus an account of genetic resistance to cadmium in one population of the oligochaete Limnodrilus hoffmeisteri concludes with the important, though unoriginal, point that the occurrence of resistance may be an effective indicator of effects on ecosystems, yet ignores the wealth of relevant material on this topic. One useful chapter describes the ecotoxicological requirements of seven pieces of legislation in the United States, including ToSCA (Toxic Substances Control Act), but with no accompanying critique. The next, final, chapter draws the obvious conclusion that we need therefore to monitor the permitted uses of chemicals, and need to be able to modify decisions in the light of experience. Despite the importance of monitoring, there is only one short chapter on the topic, and it is dominated, like many others, by a preoccupation with abstract concepts, in this instance control theory and decision analysis.

The book is not easy to use. The index is of very little help, sometimes with tens of page references per topic (58 for 'effects'). The writing is frequently ponderous, and there is considerable repetition between chapters. But those whose thinking is still influenced by the idea of a community as a supra-organism, or who are looking for an account of relevant US legislation, will find it respectively instructive or useful.

F. Moriarty, Queens Orchard, Little Eversden, Cambridge CB3 $7 \mathrm{HD}, \mathrm{UK}$, is a consultant ecotoxicologist.

- Newly published by CRC Press is Practical Handbook of Environmental Control, edited by Conrad P. Straub, a reference work intended both for students and professionals. The material, presented largely in tabular form, is divided into sections on air, water sources and quality, wastewater, solid wastes, and institutions. Price is $\$ 52.95, £ 32$ (in Britain the book is distributed by Wolfe Medical, London).
This is a great book, and an exciting book... readable, worth reading and enlightening.

Sir Karl Popper

Cosmology, Physics \& Philosophy \section{by \\ Prof Ben Gal-Or}

"a master piece. . . any good library must have a copy of this classical work."

Ind. J. Phys.

... . a tour de force. . . will be widely read and appreciated. . . a magnificent and sustained piece of work!" Sir Alen Cottrell

.. remarkable. .. challenging. . . Am. J. Phys.

Rich in thought... Strongly recommended around the world.

Chinese Academy of Sciences

2nd Printing

Springer-Verlag

175 5th Ave., N.Y., 10010

\section{FUNA-COLLAGEN}

The new collagen products for tissue cultures and related fields

FUNA-COLLAGEN is a highly purified pyrogen free collagen products manufactured with high technologies under advanced quality controls.

The following products are now available for resesrch purposes.

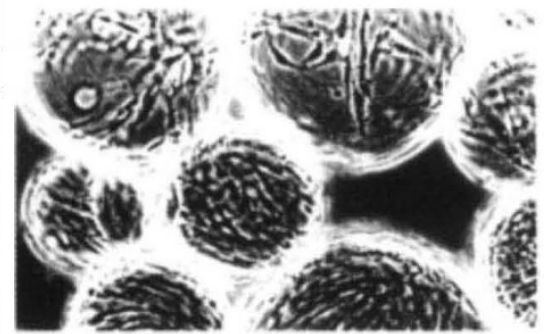

* Collagen solution

1. Acidic solution, Bovine corium pepsin solubilized type I collagen solution pH 3.0

2. Acidic solution, Bovine corium type I collagen $\mathrm{pH} 30$.

3. Neutral solution, Bovine corium pepsin solubilized type I collagen solution $\mathrm{pH} 7.4$.

* Permeable collagen membrane and device * Collagen microsphere

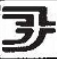

For more information write to :

FUNAKOSHI PHARMACEUTICAL CO., LTD. 2-3 Surugadai, Kanda, Chiyoda-ku, Tokyo, Japan Telephone: Tokyo 03-293-2367

Telefax : :81-3-295-5545

Telex : J28489FuNA 